\title{
STRUCTURAL AND OPTICAL STUDIES OF CdTe/ZnTe SUPERLATTICES WITH ULTRATHIN ZnTe LAYERS
}

\author{
II. Mariette, P.it. Jouneau, N.T. Pelekanos, A. Tardot, G. Feuillet \\ AND N. MAGNEA
}

\author{
CEA/CNRS Group "Microstructures de Semiconducteurs II-VI" \\ Laboratoire de Spectrométric Physique, Université J. Fourier, Grenoble \\ and \\ Département de Recherche Fondamentale sur la Matière Condensée \\ CENG, 85 X, 38041 Grenoble Cédex, France
}

\begin{abstract}
Spatially selective introduction of ultrathin $\mathrm{ZnTe}$ layers (1 to 3 monolayers) into CdTe allows the study of special superlattice structure, corresponding to a monomolecular plane-host crystal system. Particular attention is given to the strain state control of the inserted $\mathrm{ZnTe}$ monolayer. IIigh resolution electron microscopy is used to measure the local lattice distortion: the method yields the location and the total amount of $\mathrm{Zn}$ per period, and the results are compared with X-ray diffraction data. Optical properties of these superlattices are also presented. All results show the ability to control ultrathin pseudomorphic layers of ZnTe within CdTe, with limited Zn segregation, and of ligh crystalline and optical quality. In addition, they can be fitted within the framework of elasticity theory for the structural data, and of a finite quantum well model for the optical ones, even in the ultimate limit of only one nominal ZnTe monolayer.
\end{abstract}

PACS numbers: 78.66.IIf, 73.61.Ga

\section{Introduction}

High quality layer-by-layer growth of semiconductor heterostructures has made it possible to realize layered materials built up of different compounds with individual layers thicknesses down to the monomolecular scale. A particular example, of interest to this paper, is the spatially selective incorporation within semiconductor heterostructures of isoelectronic substituents of host atoms in the form of two-dimensional (2D) mono-atomic "sheets". Such isoelectronic planar perturbations are a very powerful tool to probe the intrinsic properties of heterostructures, like the wave function of the ground and excited quantum well (QW) levels [1] or the nature (spatially direct or indirect) of the exciton transition [2].

A key element in such a work is the successful incorporation and characterization of the monomolecular layer inserted into the heterostructure. It is then 
necessary to study a monomolecular plane-host crystal system which represents a new sort of superlattice (SL) structure [3]. Apart from the fabrication challenge, the realization of these SLs leads to the fundamental question: can the bulk crystal characteristics of the constituent materials be used to describe the structural and electronic propertics of these systems? For III-V lattice mismatched materials, this problem was addressed by measuring the local distortion of ultrathin InAs layers embedded in GaAs [4] and their photoluminescence properties [3]. It was proposed that the elasticity theory fails to account for the distortion of one InAs monolayer [5] and that a self-consistent pseudopotential method is necessary to calculate the energy gap in the monomolecular-plane systcm [6]. We report here on the II-VI $\mathrm{CdTe} / \mathrm{ZnTe}$ lattice mismatched system where ultrathin ZnTe layers were grown into CdTe by molecular beam epitaxy (MBE). Our approach consists in comparing three sets of experimental data, namely high resolution electron microscopy (IIREM) analysis, X-ray double diffraction and optical spectroscopy. This allows us to assess the ability to grow within $\mathrm{CdTe}$, ultrathin $2 \mathrm{D}$ pseudomorphic ZnTe layers with limited Zn segregation and of high crystalline and optical quality, and to account for the low-dimensional phenomena wich occur in such superlattice systems.

The paper is organised as follows: the sample design considerations and the experimental methods are described in Sec. 2. In Sec. 3 we present a structural study based on a IIREM image analysis which provides the local lattice distortions of the strained ZnTe monolayer plane. These results are compared with $\mathrm{X}$-ray double diffraction data and with other strained systems like InAs/GaAs and MnTe/CdTe. The optical properties (photoluminescence and photoluminescence excitation) of these CdTe/ZnTe SLs are presented in Sec. 4. The exciton ground and excited levels obtained from these data are compared to these deduced from a calculation which takes into account the interface profile inferred from the structural study. Finally, we conclude in Sec. 5.

\section{Sample description and experimental techniques}

The samples were grown by MBE on (100) oriented $\mathrm{Cd}_{1-y} \mathrm{Zn}_{y}$ Te substrates with $y=3 \%$ at $T=280^{\circ} \mathrm{C}$. They consist of binary CdTe/ZnTe strained SLs. The sample are named after $n / m$ where $n$ and $m$ are the nominal numbers of monolayers (MLs) within one SL period of respectively CdTe and $\mathrm{ZnTe}$. All samples except the $27 / 3$ one consist of 100 periods and are grown directly on the substrates, whereas the $27 / 3 \mathrm{ML}$ sample consists of only five periods and is grown on a $2 \mu \mathrm{m}$ thick CdZnTe buffer with $15 \% \mathrm{Zn}$, for reasons of strain symmetrization as will be explained next.

For all samples, the relative layer thicknesses, the total number of periods and the $\mathrm{Zn}$ composition of the buffer layer were adjusted in order to ensure pseudomorphic growth of the SL, i.e. the in-plane lattice parameter of each layer within the $\mathrm{SL}$ is imposed by the lattice parameter of the $\mathrm{Cd}_{1-y} \mathrm{Zn}_{y} \mathrm{Te}$ buffer (or of the substrate in the absence of a buffer). Pseudomorphic growth presupposes the absence of any strain relaxation in the SL-region of the sample in the form of misfit dislocations which would severely degrade the crystalline and optical qualities. In order to achieve this, two criteria must be fulfilled. (i) All individual layer thick- 
nesses in the SL have to be kept below their respective critical thickness $t_{\mathrm{c}}$, which is defined as the thickness of the strained layer above which the elastic energy exceeds the dislocation generation threshold. Given that the $\mathrm{CdTe} / \mathrm{ZnTe}$ system exhibits a large lattice mismatch $(6.4 \%)$, the critical thickness $t_{\mathrm{c}}$ over which such a relaxation occurs is very small. It was recently shown that $t_{\mathrm{c}}$ varies for the $(001)$ growth orientation from $t_{\mathrm{c}} \approx 4000 \AA$ for CdTe grown on $\mathrm{Cd}_{0.92} \mathrm{Zn}_{0.03} \mathrm{Te}$ to $t_{\mathrm{c}} \approx 17 \AA(5 \mathrm{ML})$ for CdTe on ZnTe [7]. (ii) The average strain of the SL has to be close to zero (symmetrized strain state). When this condition is fulfilled, i.e. the average lattice parameter of the SL along the growth axis $a_{\mathrm{SL}}$ is the same as the lattice parameter of the strain imposing layer $a_{0}$, then whatever the total thickness of the SL pseudomorphic growth is maintained [8]. This is possible when the lattice parameter of the strain imposing layer lies in between that of the two components of the SL such as, for example, when growing a CdTe/ZnTe SL directly on a $\mathrm{Cd}_{0.97} \mathrm{Zn}_{0.03}$ Te substrate: in this particular case, the CdTe layers are in weak compression and the $\mathrm{ZnTe}$ oncs in strong expansion. The layer thickness ratio of $\mathrm{CdTe}$ to $\mathrm{ZnTe}$ (and the $\mathrm{Zn}$ composition of the buffer layer for the $27 / 3$ sample) was adjusted to obtain the symmetrized strain state. Thus, we were able to obtain $1 \mu \mathrm{m}$ thick (100 periods) SLs with no strain relaxation in spite of the large lattice mismatch of the SL constituent materials.

Structural information about these heterostructures was obtained by X-ray diffraction and transmission electron microscopy (TEM). The X-ray measurements were performed on a double diffraction system equipped with an InSb (001) monochromator in the vicinity of the (004) reflection. For TEM analysis we used a JEOL 4000EX and 200CX electron microscope. Cross-sectional (110) oriented TEM foils were prepared by conventional $\mathrm{Ar}^{+}$ion milling techniques. The local distortions of the lattice at or near the $\mathrm{Zn}$ Te layer are deduced from an image processing using the same method as Bierwolf et al. [4]: from a reference area on the IIREM image, an ideal lattice is extrapolated on the whole image. Then the displacements between each point of the ideal lattice and each experimental atom-pair position are measured, and the local distortion between two (002) atomic planes is deduced.

The optical measurements were performed out in a pumped liquid He immersion dewar at $1.8 \mathrm{~K}$. The photoluminescence (PL) measurements were performed with an $\mathrm{Ar}^{+}$laser whereas, for photoluminescence excitation (PLE) studies, the samples were excited by a tunable Ti:sapplire laser. The circular polarization of PLE spectra (PPLE) was measured using an electrooptic modulator. In such an optical pumping experiment one excites the sample with circularly polarized light and measures the degree of circular polarization of the emission. The principle and the details of this technique which allows us to distinguish the light hole from the heavy hole character of the optical transitions are given in Ref. [9].

\section{Structural study}

Different $\mathrm{CdTe} / \mathrm{ZnTe}$ samples with $\mathrm{ZnTe}$ varying between 1 and 3 monolayers were observed by TEM in (110) cross-section. This analysis was performed either in dark field image mode with a (200) diffracted beam (Fig. 1a) or in high resolution mode (IIRTEM) (Fig. 1b). In Fig. 1a the strong chemical contrast be- 
tween CdTe and ZnTe allows us to distinguish the two nominal ZnTe MLs (white strips) buried in CdTe (dark background). Within the limits of this experimental technique (a TEM image averages a few liundreds of $\AA$ of material along the (110) direction) the ultrathin $\mathrm{ZnTe}$ layers are quite uniform and without any discontinuity. We also notice the absence of interface dislocations attesting for pseudomorphic growth throughout the heterostructure. In Fig. 1b the HRTEM image for the 57/2 reveals the pseudomorphic growth of the SL layers. The analysis of the HRTEM images provides us with detailed structural information especially about the local distortion between each plane induced by the tetragonally strained ZnTe layers. Figure 2 shows the result of the distortion analysis [10] on three samples. Even though the distortions are mainly localised on one, two or three monolayers, there are still some residual distortions extending on a few planes on either side of the inserted layers. Furthermore, the maximum observed distortion is lower than the expected one. This indicates either the presence of non-abrupt interfaces, resulting from interfacial roughness and also possibly from some $\mathrm{Zn}$ segregation during
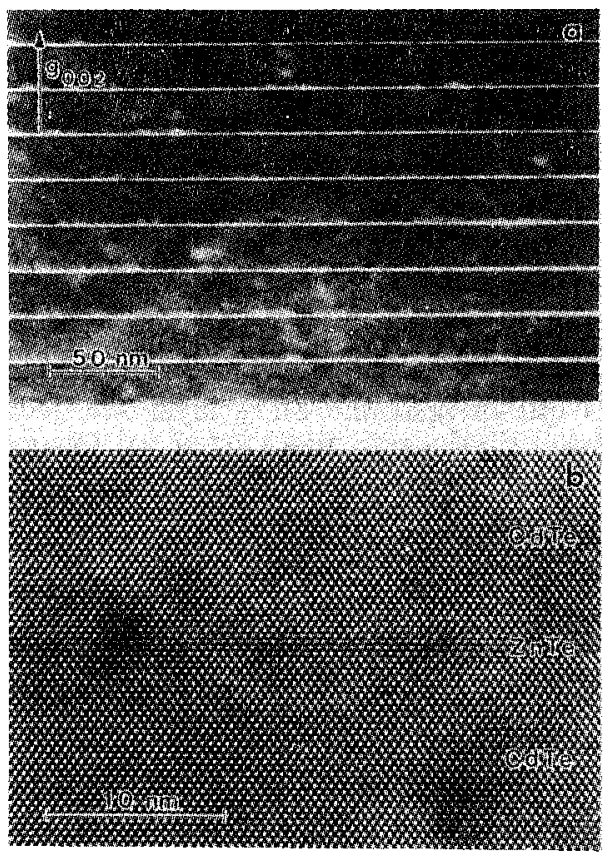

Fig. 1. (a) Dark field micrograph $(g=002)$ of the $57 / 2$ sample. ZnTe layers appear in white, CdTe in dark. (b) High resolution TEM image of the $57 / 2$ sample observed in [110] cross-section.

growth, or a departure from the predictions of continuum elasticity theory in the strain state of such ultrathin ZnTe layers.

To answer this question, the local distortion is integrated along the (001) growth axis within one SL period in order to obtain the net translation per period 


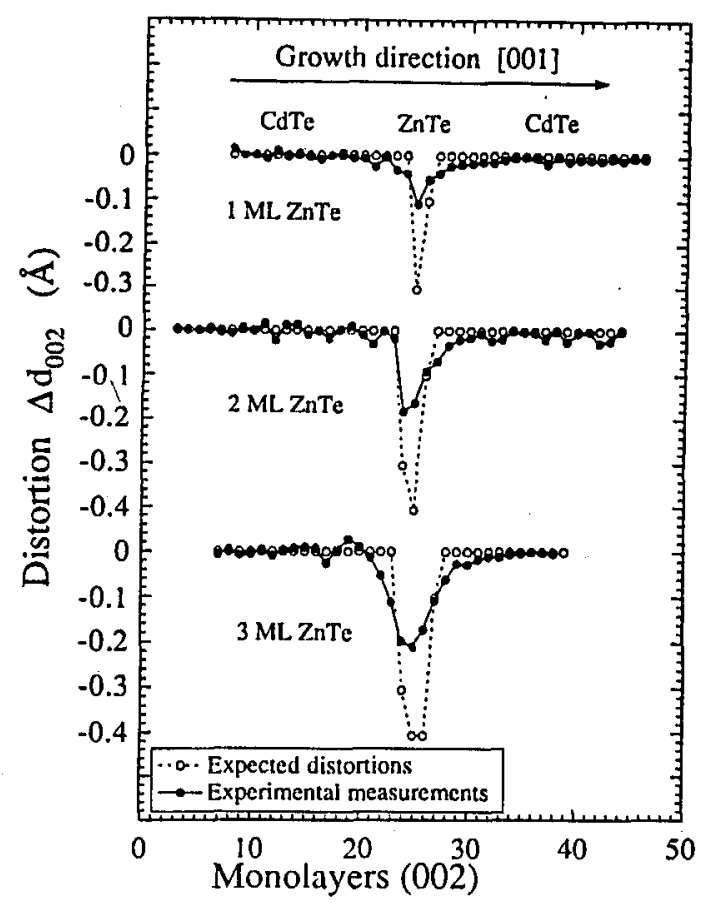

Fig. 2. Local distortion analysis results on HREM images of CdTe/ZnTe samples 27/1, $57 / 2$ and $88 / 3$.

of the CdTe lattice induced by the highly strained CdTe layers. The results are reported in Fig. 3 and compared with the ones obtained for $\mathrm{MnTe} / \mathrm{CdTe}$ [10] and InAs/GaAs $[4,5]$. The former system has a lattice mismatch of $6.2 \%$ similar to the $\mathrm{ZnTe} / \mathrm{CdTe}$ one which allows to insert only a few MLs of InAs planes into $\mathrm{GaAs}$ without relaxation occurring. For $\mathrm{MnTe} / \mathrm{CdTe}$ SLs, the lattice mismatch is smaller $(2.2 \%)$ and then the growth of MnTe pseudomorphic layers in CdTe is possible for MnTe thicknesses reaching up to $40 \mathrm{MLs}$ [10]. For all 3 cases the total lattice distortion is plotted as a function of the nominal number of inserted monolayers. It is compared with the values expected for a pseudomorphic layer assuming the elasticity theory is valid (full line), and for a completely relaxed inserted layer (dashed line). Given the uncertainty on the amount of $\mathrm{Zn}$ atoms incorporated during growth, no breakdown of the continuum elasticity theory is necessary to explain our results unlike the conclusion reached by the workers of Ref. [5] for the strain state of one InAs ML embedded in GaAs. However, the local distortion analysis shows that, even though the lattice mismatch strain is mainly accomodated in the $\mathrm{ZnTe}$ planes (within $\approx 70 \%$ ), there is still some residual strain extending over 3 to $5 \mathrm{MLs}$ after the last ZnTe plane location. This is possibly due to $\mathrm{Zn}$ segregation during growth of the $\mathrm{ZnTe}$ thin layers which leads to an asymmetric repartition of the $\mathrm{Zn}$ atoms and hence to the observed asymmetric 

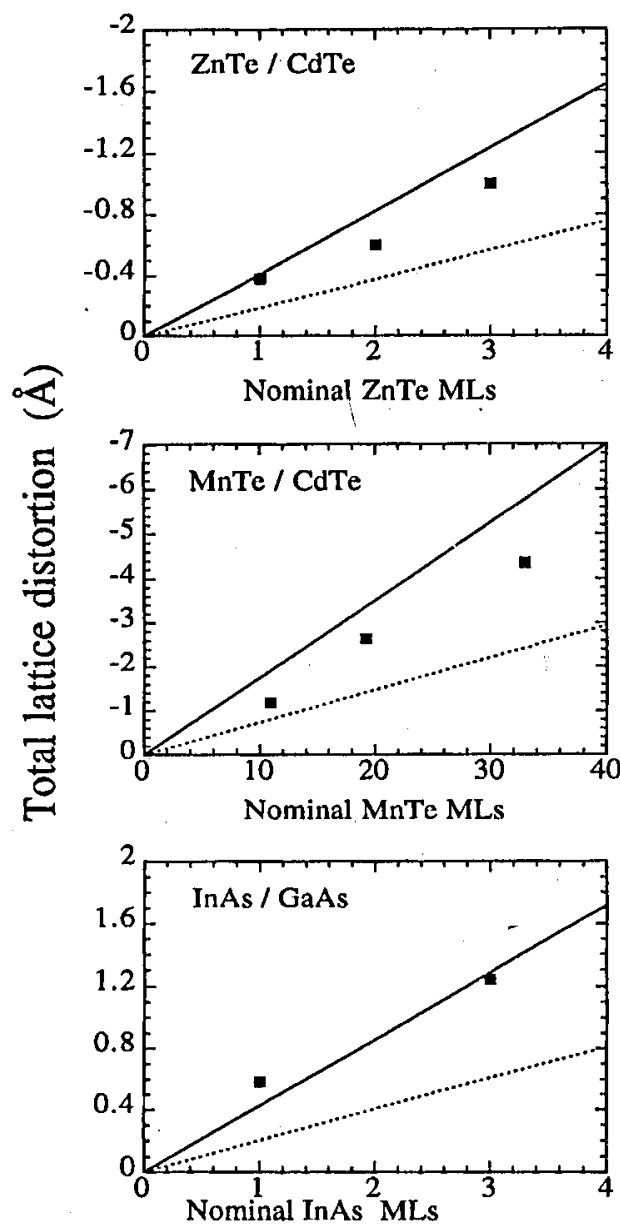

Fig. 3. Total lattice distortion deduced from HREM image analysis for three different systems $\mathrm{CdTe} / \mathrm{ZnTe}, \mathrm{CdTe} / \mathrm{MnTe}$ and $\mathrm{InAs} / \mathrm{GaAs}$ as a function of the number of nominally inserted monolayers (MLs). These experimental data (full squares) are compared to the values expected for a completely relaxed inserted layer (dashed lines) and for a pseudomorphic one assuming the validity of the elasticity theory (full lines). The data for InAs/GaAs are deduced from Refs. [4, 5].

strain profile. If we translate, within the elasticity framework, the strain profile into a $\mathrm{Zn}$-concentration profile, we can estimate, as for instance for sample 57/2, the amount of $\mathrm{Zn}$ at its nominal place to be about $60 \%$ and a typical length over which $90 \%$ of the $\mathrm{Zn}$ atoms are redistributed of the order of $4 \mathrm{MLs}$.

It is interesting to note that the asymmetry of the strain profile supports a segregation mechanism rather than a during-growth interdiffusion process which 
should result in more symmetric profiles. Moreover, annealing studies [11] on $\mathrm{CdTe} / \mathrm{ZnTe}$ and $\mathrm{CdTe} / \mathrm{CdZnTe}$ systems yields diffusion coefficients of ZnTe in $\mathrm{CdTe}$ or $(\mathrm{Cd}, \mathrm{Zn}) \mathrm{Te}$ at the growth temperature $T=280^{\circ} \mathrm{C}$ of the order of $10^{-22} \mathrm{~cm}^{2} / \mathrm{s}$. This corresponds, for an one-hour long growth, to a diffusion length of less than $0.1 \AA$ indicating that interdiffusion is negligible in our case.

An alternative way was used to analyse the presence of $\mathrm{Zn}$ atoms within the SL period: high resolution X-ray double diffraction (HRXRD). Owing to the large lattice mismatch strain accomodate mainly in the $\mathrm{ZnTe}$ layer as well as the larger elastic constants of $\mathrm{ZnTe}$ compared to $\mathrm{CdTe}, \mathrm{X}$-ray diffraction is very sensitive (sub ML resolution) to the number of ${ }_{71}$ Te MLs present in the SL. Figure 4 represents the full spectrum of IIRXRD for various CdTe/ZnTe samples [12]. From the relative spacing of the satellite peaks, the exact SL period is determined. The

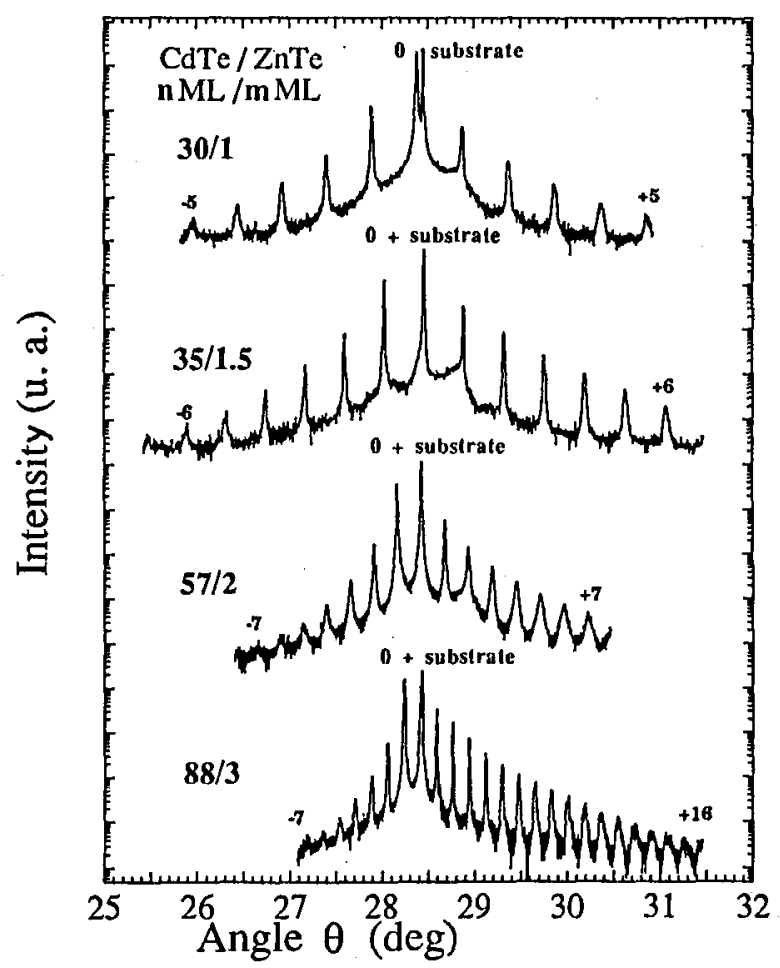

Fig. 4. (004) X-ray double diffraction spectra of pseudomorphic CdTe/ZnTe superlattices.

zeroth-order peak, corresponding to the average lattice constant of the SL along the growth axis is almost concident with the substrate peak as expected from the SL-parameter design (strain symmetrization). The exact angular position of the zeroth-order peak together with the satellite ones yields the total amount of $\mathrm{Zn}$ per period. Some values are reported in Table and compared with the ones 
given by IIREM. A very good agreement is obtained between the two sets of values, confirming the validity of our method. In all cases, the total amount of $\mathrm{Zn}$ inserted during the growth was found to be lower than the one expected from the growth rate calibrations. We would like to point out that a unique advantage of such HREM study is to yield the location of $\mathrm{Zn}$ atoms within a period.

TABLE

Comparison of the total amount of $\mathrm{Zn}$ per period measured by IIREM and HRXRD.

\begin{tabular}{c|c|c|c}
\hline \hline $\begin{array}{c}\text { SLs sample } \\
\mathrm{CdTe} / \mathrm{ZnTe} \\
n \mathrm{ML} / m \mathrm{ML}\end{array}$ & $\begin{array}{c}\text { Measured period } \\
\text { (IIREM) }\end{array}$ & $\begin{array}{c}\text { Total amount of } \\
\text { Zn per period } \\
\text { (IIREM) }\end{array}$ & $\begin{array}{c}\text { Total amount of } \\
\text { Zn per period } \\
\text { (IIRXRD) }\end{array}$ \\
\hline $27 / 1$ & $27.5 \pm 0.5 \mathrm{ML}$ & $0.86 \pm 0.05 \mathrm{ML}$ & $0.8 \pm 0.1 \mathrm{ML}$ \\
$57 / 2$ & $59 \pm 0.5 \mathrm{ML}$ & $1.38 \pm 0.05 \mathrm{ML}$ & - \\
$88 / 3$ & - & $2.32 \pm 0.05 \mathrm{ML}$ & $2.2 \pm 0.1 \mathrm{ML}$
\end{tabular}

Finally, the $\mathrm{Zn}$ concentration profile deduced from HREM at the interface can be used to simulate the expected IIRXRD profile. Such non-abrupt interfaces induce a relative change in the satellite line intensities as compared to the zeroth-order peak one, corresponding qualitatively to the experimental data [12].

\section{Optical properties}

The PL spectra of three CdTe/ZnTe SL samples with respectively 1,2 and 3 $\mathrm{ZnTe}$ MLs are plotted in Fig. 5. The lines denoted by $\mathrm{e}_{1} \mathrm{~h}_{1}$ correspond to intrinsic free heavy hole-exciton transition and the ones by $Y$ to transitions of extrinsic type. The substrate contribution is also marked (sub.). Concerning the optical quality of these binary SLs, several points are worth noting: (i) the PL spectra are dominated by intrinsic free-exciton recombination. This was verified by comparing PL with reflectivity and PLE spectra and measuring a negligible Stokes shift $(\approx 0.2 \mathrm{meV})$. (ii) Another optical quality criterion is the sharpness of the $\mathrm{e}_{1} \mathrm{~h}_{1}$ lines. The full width at half maximum (FWIIM) is approximately $2 \mathrm{meV}$. This is strikingly different from all previously published optical data [13-16] on $\mathrm{CdTe} / \mathrm{ZnTe}$ SLs where the FWIIM of the PL lines ranges between 20 and $50 \mathrm{meV}$. This order of magnitude improvement is most probably due to the fact that no strain relaxation has occurred in our SLs, as elaborated above. In contrast, the samples studied in the previous references consisted of structures supposed to be in a strain-symmetrized state after some initial relaxation (free-standing SLs). Such relaxation, however, is at the origin of a high defects density and inhomogeneities in the SL-region and accounts for the large Stokes shifts and linewidths, observed until now. We would like also to point out the effect of the ultrathin $\mathrm{ZnTe}$ layers in blueshifting the $e_{1} h_{1}$ lines. For comparison, the black arrow points at the free exciton energy position of bulk CdTe. The upward white arrows indicate the calculated energy positions of the $e_{1} h_{1}$ SL lines within the theoretical model mentioned 


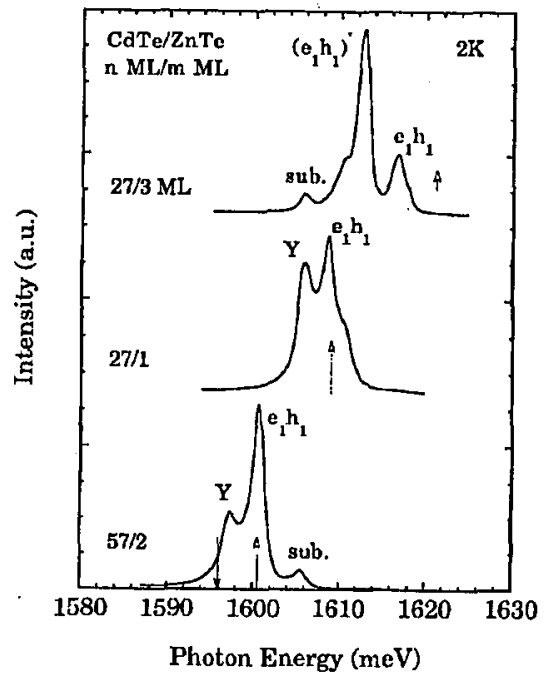

Fig. 5. PL spectra from different $\mathrm{CdTe} / \mathrm{ZnTe}$ SLs. The intrinsic heavy hole exciton transitions are denoted by $\mathrm{e}_{1} \mathrm{~h}_{1}$ and the extrinsic lines by $Y$, where visible. Similarly, the contribution from the substrate is indicated (sub.). The upward white arrows indicate the calculated $e_{1} h_{1}$ energy positions and the downward black arrow shows for comparison the bulk CdTe free exciton position.

later [17]. In general, a good agreement between calculation and experiment is obtained. The $\left(e_{1} h_{1}\right)^{\prime}$ transition for the small number of periods $27 / 3$ sample is due to the fact that the first and the last SL periods are not equivalent to the intermediate ones.

Figure 6 displays various optical spectra for a $57 / 2$ SL sample. Besides the PL measurement which provides the energy position of the exciton ground state $e_{1} h_{1}$, the PLE spectrum exhibits the excited states $e_{1} l_{1}$ and $e_{1} h_{2}$ whereas the circular polarized PLE allows to distinguish between the light and heavy hole excitons which are involved in the different transitions. The experimental exciton transition energies are compared to a calculational model [17]. To account for the exciton binding energies, this envelope wave function calculation includes when solving for the Schrödinger equation, an effective potential for the holes whichi is the sum of the quantum well potential and the Coulomb interaction. The potential profile is assumed to be either abrupt with a ZnTe 2 ML nominal thickness, or smooth with a Zn-concentration profile deduced from IIREM (see Sec. 3). The results are marked in Fig. 6 by the full line arrows for the first case and the dashed ones for the second case. The difference between the two approaches is the relative energy position of $e_{1} l_{1}$ (white arrows) as compared to $e_{1} h_{1}$ (black arrows). This result seems to confirm that (i) a finite quantum well model using, for the band parameters, the values of CdTe and ZnTe compounds can account for the energy levels observed in these $\mathrm{CdTe} / \mathrm{ZnTe} \mathrm{SLs}$, (ii) the $\mathrm{Zn}$ concentration profile deduced from HREM allows us to obtain better agreement with the optical data than the nominal abrupt one. 


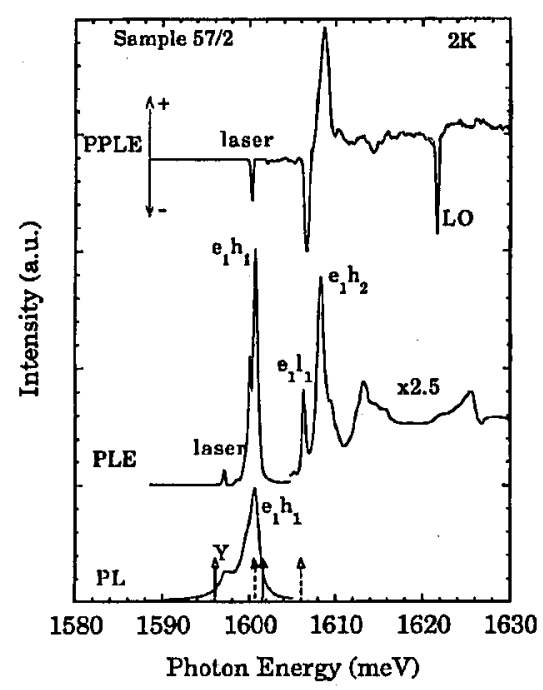

Fig. 6. Comparison of PL, PLE and circular polarized PLE (PPLE) spectra for CdTe/ZnTe sample 57/2. The main exciton transitions $e_{1} h_{1}, e_{1} l_{1}$ and $e_{1} l_{2}$ are indicated. Full lines arrows represent the calculated exciton transition energies of $e_{1} h_{1}$ (black) and $\mathrm{e}_{1} \mathrm{l}_{1}$ (white) respectively with a $2 \mathrm{ML} \mathrm{ZnTe}$ abrupt interface. Dashed line arrows represent the calculated exciton transition energies with a $\mathrm{Zn}$ concentration profile deduced from HREM.

\section{Conclusion}

In summary, we have carefully characterized the incorporation of ultrathin ZnTe layers in CdTe. Specifically, TEM and X-ray diffraction studies on binary $\mathrm{Cd} \mathrm{Te} / \mathrm{ZnTe}$ superlattices showed that pseudomorphic and dislocation-free $\mathrm{CdTe} / \mathrm{ZnTe}$ heteroepitaxy is possible, with a monolayer growth control. Moreover, the local distortion in each monomolecular plane is deduced suggesting the presence of non-abrupt interfaces. Assuming the validity of the elasticity theory in such ultrathin layers, these strain data are translated into a $\mathrm{Zn}$ concentration profile and the total amount of $\mathrm{Zn}$ per SL period can be quantified. These results are in agreement with the ones given by HRXRD. In addition, optical spectroscopic data exhibit sharp free exciton recombination lines, indicating an optimal optical quality for this heterostructure system. All these experimental results can be modelled within the framework of elasticity theory for the structural data, and of a finite QW model for the optical ones, even in the ultimate limit of only one nominal ZnTe monolayer.

Finally, planar ZnTe isoelectronic perturbations can be used to gain direct microscopic insight into the exciton states in strained $\mathrm{CdTe} /(\mathrm{Cd}, \mathrm{Zn}) \mathrm{Te}$ superlattices. This system has a mixed type band configuration, namely the electrons and heavy holes are confined in the CdTe layers whereas the light holes are localized in the CdZnTe ones. Then the nature (spatially direct or indirect) of the heavy 
hole and light hole exciton can be probed by inserting one $\mathrm{ZnTe}$ monolayer either in the $(\mathrm{Cd}, \mathrm{Zn}) \mathrm{Te}$ barrier or in the CdTe well [18].

\section{References}

[1] J.Y. Marzin, J.M. Gérard, Phys. Rev. Lett. 62, 2172 (1989).

[2] J.M. Gérard, J.Y. Marzin, Phys. Rev. B 40, 6450 (1989).

[3] M. Sato, Y. Horikoshi, J. Appl. Phys. 66, 851 (1989).

[4] R. Bierwolf, M. Hohenstein, O. Brandt, G.E. Crook, K. Ploog, Ultramicroscopy 49, 273 (1993).

[5] O. Brandt, K. Ploog, R. Bierwolf, M. Hohenstein, Phys. Rev. Lett. 68, 1339 (1992).

[6] K. Shiraishi, E. Yamaguchi, Phys. Rev. B 42, 3064 (1990).

[7] P.H. Jouneau, J. Cibert, G. Feuillet, R.E. Mallard, K. Saminadayar, S. Tatarenko, Le Si Dang, Inst. Phys. Conf. Ser. 117, 623 (1991).

[8] E. Kasper, H. Kibbel, HI. Jorke, H. Brugger, E. Friess, G. Abstreiter, Phys. Rev. B 38, 3599 (1988); A. Ponchet, G. Lentz, H. Tuffigo, N. Magnea, H. Mariette, P. Gentile, J. Appl. Phys. 68, 6229 (1990).

[9] C. Weisbuch, R.C. Miller, R. Dingle, A.C. Gossard, W. Wiegmann, Solid State Commun. 37, 219 (1981).

[10] P.H. Jouneau, A. Tardot, G. Feuillet, H. Mariette, J. Cibert, in: Proc. 8th Oxford Conf. on Microscopy of Semiconducting Materials, 1993, to be published in Inst. Phys. Conf. Ser.

[11] A. Hamoudi, E. Ligeon, K. Saminadayar, J. Cibert, Le Si Dang, S. Tatarenko, Appl. Phys. Lett. 60, 2797 (1992); N. Magnea, H. Mariette, N. Pelekanos, A. Tardot, Mater. Sci. Eng. B 16, 71 (1993).

[12] A. Tardot, Ph.D. Thesis, University Joseph Fourier, Grenoble 1993.

[13] R.H. Miles, G.Y. Wu, M.B. Jonhson, T.L. McGill, J.P. Faurie, S. Sivananthan, Appl. Phys. Lett. 48, 1383 (1986).

[14] Y. Hefetz, D. Lee, A.V. Nurmikko, S. Sivananthan, Y. Chu, J.P. Faurie, Phys. Rev. B 34, 4423 (1987).

[15] H. Mathieu, J. Allegre, A. Chatt, P. Lefebvre, J.P. Faurie, Phys. Rev. B 38, 7740 (1988).

[16] B. Gil, D.J. Dunstan, J. Calatayud, H. Mathieu, J.P. Faurie, Phys. Rev. B 40, 5522 (1989).

[17] P. Peyla, Ph.D. Thesis, Université Joseph Fourier, Grenoble 1992.

[18] N.T. Pelekanos, P. Peyla, Le Si Dang, H. Mariette, P.H. Jouneau, A. Tardot, N. Magnea, accepted in Phys. Rev. B, 1993. 